\title{
Some properties of four-dimensional Walker manifolds
}

\author{
Abdoul Salam Diallo ${ }^{1}$, Fortune Massamba ${ }^{2}$ \\ ${ }^{1}$ School of Mathematics, Statistics and Computer Science, University of KwaZulu-Natal, Private Bag X01, Scottsville, South Africa \\ and Université Alioune Diop de Bambey UFR SATIC, Département de Mathématiques, Bambey, Senegal \\ ${ }^{2}$ School of Mathematics, Statistics and Computer Science, University of KwaZulu-Natal, Private Bag X01, Scottsville, South Africa
}

Received: 29 December 2016, Accepted: 12 April 2017

Published online: 26 August 2017.

\begin{abstract}
In this paper, we investigate geometric properties of some curvature tensors of a four-dimensional Walker manifold. Some characterization theorems are also obtained.
\end{abstract}

Keywords: Einstein metric, locally symmetry, locally conformally flat, Walker metrics.

\section{Introduction}

A Walker $n$-manifold is a pseudo-Riemannian manifold, which admits a field of parallel null $r$-planes, with $r \leq \frac{n}{2}$. The canonical forms of the metrics were investigated by A. G. Walker [10]. Of special interest are the even-dimensional Walker manifolds $(n=2 m)$ with fields of parallel null planes of half dimension $(r=m)$.

It is known that Walker metrics have served as a powerful tool of constructing interesting indefinite metrics which exhibit various aspects of geometric properties not given by any positive definite metrics. Among these, the significant Walker manifolds are examples of the non-symmetric and non-homogeneous Osserman manifolds [2,3]. Recently, it was shown $[4,6,7]$ that the Walker 4-manifolds of neutral signature admit a pair comprising an almost complex structure and an opposite almost complex structure, and that Petean's nonflat indefinite Kahler-Einstein metric on a torus was obtained as an example of a Walker 4-manifold. Moreover, Banyaga and Massamba derived in [1] a Walker metric when studying the non-existence of certain Einstein metrics on some symplectic manifolds.

Our purpose is to study restricted 4-Walker metrics by focusing on their curvature properties. The main results of this paper are the characterization of Walker metrics which are Einstein, locally symmetric Einstein and locally conformally flat. The paper is organized as follows. In section 2, we recall some basic facts about Walker metrics by explicitly writing its Levi-Civita connection and the curvature tensor. Walker metrics which are Einstein are investigated in section 3 (Theorem 1). In section 4, we study the Walker metrics which are locally symmetric Einstein (Theorem 2). Finally, we discuss in section 5, the conformally locally flat property of Walker metric (Theorem 3).

\section{The canonical form of a Walker metric}

Let $M$ be a pseudo-Riemannian manifold of signature $(n, n)$. We suppose given a splitting of the tangent bundle in the form $T M=\mathscr{D}_{1} \oplus \mathscr{D}_{2}$ where $\mathscr{D}_{1}$ and $\mathscr{D}_{2}$ are smooth subbundles which are called distribution. This define two complementary projection $\pi_{1}$ and $\pi_{2}$ of $T M$ onto $\mathscr{D}_{1}$ and $\mathscr{D}_{2}$. We say that $\mathscr{D}_{1}$ is parallel distribution if $\nabla \pi_{1}=0$. 
Equivalently this means that if $X_{1}$ is any smooth vector field taking values in $\mathscr{D}_{1}$, then $\nabla X_{1}$ again takes values in $\mathscr{D}_{1}$. If $M$ is Riemannian, we can take $\mathscr{D}_{2}=\mathscr{D}_{1}^{\perp}$ to be the orthogonal complement of $\mathscr{D}_{1}$ and in that case $\mathscr{D}_{2}$ is again parallel. In the pseudo-Riemannian setting, $\mathscr{D}_{1} \cap \mathscr{D}_{2}$ need not be trivial. We say that $\mathscr{D}_{1}$ is a null parallel distribution if $\mathscr{D}_{1}$ is parallel and the metric restricted to $\mathscr{D}_{1}$ vanish identically. Manifolds which admit null parallel distribution are called Walker manifolds.

A neutral $g$ on an 4-manifold $M$ is said to be a Walker metric if there exists a 2-dimensional null distribution $\mathscr{D}$ on $M$ which is parallel with respect to $g$. From Walker theorem [10], there is a system of coordinates $\left(u_{1}, u_{2}, u_{3}, u_{4}\right)$ with respect to which $g$ takes the local canonical form

$$
\left(g_{i j}\right)=\left(\begin{array}{cc}
0 & I_{2} \\
I_{2} & B
\end{array}\right),
$$

where $I_{2}$ is the $2 \times 2$ identity matrix and $B$ is a symmetric $2 \times 2$ matrix whose the coefficients are the functions of the $\left(u_{1}, \cdots, u_{4}\right)$. Note that $g$ is of neutral signature $(++--)$ and that the parallel null 2-plane $\mathscr{D}$ is spanned locally by $\left\{\partial_{1}, \partial_{2}\right\}$, where $\partial_{i}=\frac{\partial}{\partial_{i}}, i=1,2,3,4$.

Let $M_{a, b, c}:=\left(\mathscr{O}, g_{a, b, c}\right)$, where $\mathscr{O}$ be an open subset of $\mathbb{R}^{4}$ and $a, b, c \in \mathscr{C}^{\infty}(\mathscr{O})$ be smooth functions on $\mathscr{O}$, then

$$
\left(g_{a, b, c}\right)_{i j}=\left(\begin{array}{cccc}
0 & 0 & 1 & 0 \\
0 & 0 & 0 & 1 \\
1 & 0 & a & c \\
0 & 1 & c & b
\end{array}\right)
$$

where $a, b$ and $c$ are functions of the $\left(u_{1}, \cdots, u_{4}\right)$. We denote, $h_{i}=\frac{\partial h\left(u_{1}, \cdots, u_{4}\right)}{\partial u_{i}}$ and $h_{i ; j}=\frac{\partial h\left(u_{1}, \cdots, u_{4}\right)}{\partial u_{i} \partial u_{j}}$ for any function $h\left(u_{1}, \cdots, u_{4}\right)$. In [3], Einsteinian, Osserman or locally conformally flat Walker manifolds were investigated in the restricted form of metrics when $c\left(u_{1}, u_{2}, u_{3}, u_{4}\right)=0$. In this paper, following [3], we consider the specific Walker metrics on a 4dimensional manifold with

$$
a=a\left(u_{1}, u_{2}\right), \quad b=b\left(u_{1}, u_{2}\right) \quad \text { and } \quad c=c\left(u_{1}, u_{2}\right),
$$

and investigate conditions for a Walker metric (1) to be Einsteinian, locally symmetric Einstein and locally conformally flat.

A straightforward calculation show that the Levi-Civita connection of a Walker metric (1) is given by

$$
\begin{aligned}
\nabla_{\partial_{1}} \partial_{3} & =\frac{1}{2} a_{1} \partial_{1}+\frac{1}{2} c_{1} \partial_{2}, \quad \nabla_{\partial_{1}} \partial_{4}=\frac{1}{2} c_{1} \partial_{1}+\frac{1}{2} b_{1} \partial_{2}, \\
\nabla_{\partial_{2}} \partial_{3} & =\frac{1}{2} a_{2} \partial_{1}+\frac{1}{2} c_{2} \partial_{2}, \quad \nabla_{\partial_{2}} \partial_{4}=\frac{1}{2} c_{2} \partial_{1}+\frac{1}{2} b_{2} \partial_{2}, \\
\nabla_{\partial_{3}} \partial_{3} & =\frac{1}{2}\left(a a_{1}+c a_{2}\right) \partial_{1}+\frac{1}{2}\left(b a_{2}+c a_{1}\right) \partial_{2}-\frac{1}{2} a_{1} \partial_{3}-\frac{1}{2} a_{2} \partial_{4} \\
\nabla_{\partial_{3}} \partial_{4} & =\frac{1}{2}\left(a c_{1}+c c_{2}\right) \partial_{1}+\frac{1}{2}\left(b c_{2}+c c_{1}\right) \partial_{2}-\frac{1}{2} c_{1} \partial_{3}-\frac{1}{2} c_{2} \partial_{4} \\
\nabla_{\partial_{4}} \partial_{4} & =\frac{1}{2}\left(a b_{1}+c b_{2}\right) \partial_{1}+\frac{1}{2}\left(b b_{2}+c b_{1}\right) \partial_{2}-\frac{1}{2} b_{1} \partial_{3}-\frac{1}{2} b_{2} \partial_{4} .
\end{aligned}
$$


From relations above, after a long but straightforward calculation we get that the nonzero components of the $(0,4)$ curvature tensor of any Walker metric (1) are determined by

$$
\begin{aligned}
& R_{1313}=\frac{1}{2} a_{1 ; 1}, \quad R_{1314}=\frac{1}{2} c_{1 ; 1}, \quad R_{1323}=\frac{1}{2} a_{1 ; 2}, \quad R_{1324}=\frac{1}{2} c_{1 ; 2}, \\
& R_{1334}=\frac{1}{4}\left(a_{2} b_{1}-c_{1} c_{2}\right), \quad R_{1414}=\frac{1}{2} b_{1 ; 1}, \quad R_{1423}=\frac{1}{2} c_{1 ; 2}, \quad R_{1424}=\frac{1}{2} b_{1 ; 2}, \\
& R_{1434}=\frac{1}{4}\left(c_{1}^{2}-a_{1} b_{1}+b_{1} c_{2}-b_{2} c_{1}\right), \quad R_{2323}=\frac{1}{2} a_{2 ; 2}, \quad R_{2324}=\frac{1}{2} c_{2 ; 2}, \\
& R_{2334}=\frac{1}{4}\left(-c_{2}^{2}+a_{2} b_{2}+a_{1} c_{2}-a_{2} c_{1}\right), \quad R_{2424}=\frac{1}{2} b_{2 ; 2}, \quad R_{2434}=\frac{1}{4}\left(-a_{2} b_{1}+c_{1} c_{2}\right), \\
& R_{3434}=\frac{1}{4}\left(a c_{1}^{2}+b c_{2}^{2}-a a_{1} b_{1}-c a_{1} b_{2}-c a_{2} b_{1}-b a_{2} b_{2}+2 c c_{1} c_{2}\right) .
\end{aligned}
$$

Next, let $\rho(X, Y)=\operatorname{trace}\{Z \longrightarrow R(X, Z) Y\}$ and $S c=\operatorname{tr}(\rho)$, be the Ricci tensor and the scalarcurvature respectively. Then from (2) we have

$$
\begin{aligned}
& \rho_{13}=\frac{1}{2}\left(a_{1 ; 1}+c_{1 ; 2}\right), \quad \rho_{14}=\frac{1}{2}\left(b_{1 ; 2}+c_{1 ; 1}\right), \\
& \rho_{23}=\frac{1}{2}\left(a_{1 ; 2}+c_{2 ; 2}\right), \quad \rho_{24}=\frac{1}{2}\left(b_{2 ; 2}+c_{1 ; 2}\right), \\
& \rho_{33}=\frac{1}{2}\left(-c_{2}^{2}+a_{1} c_{2}+a_{2} b_{2}-a_{2} c_{1}+a a_{1 ; 1}+2 c a_{1 ; 2}+b a_{2 ; 2}\right), \\
& \rho_{34}=\frac{1}{2}\left(-a_{2} b_{1}+c_{1} c_{2}+a c_{1 ; 1}+2 c c_{1 ; 2}+b c_{2 ; 2}\right), \\
& \rho_{44}=\frac{1}{2}\left(-c_{1}^{2}+a_{1} b_{1}-b_{1} c_{2}+b_{2} c_{1}+a b_{1 ; 1}+2 c b_{1 ; 2}+b b_{2 ; 2}\right)
\end{aligned}
$$

and

$$
S c=\sum_{i, j=1}^{4} g^{i j} \rho_{i j}=a_{1 ; 1}+b_{2 ; 2}+2 c_{1 ; 2}
$$

The nonzero components of the Einstein tensor $G_{i j}=\rho_{i j}-\frac{S c}{4} g_{i j}$ are given by

$$
\begin{aligned}
G_{13} & =\frac{1}{4} a_{1 ; 1}-\frac{1}{4} b_{2 ; 2}, \quad G_{14}=\frac{1}{2} c_{1 ; 1}+\frac{1}{2} b_{1 ; 2}, \\
G_{23} & =\frac{1}{2} a_{1 ; 2}+\frac{1}{2} c_{2 ; 2}, \quad G_{24}=\frac{1}{4} b_{2 ; 2}-\frac{1}{4} a_{1 ; 1}, \\
G_{33} & =\frac{1}{4} a a_{1 ; 1}+c a_{1 ; 2}+\frac{1}{2} b a_{2 ; 2}-\frac{1}{2} a_{2} c_{1}+\frac{1}{2} a_{1} c_{2}+\frac{1}{2} a_{2} b_{2}-\frac{1}{2} c_{2}^{2}-\frac{1}{2} a c_{1 ; 2}-\frac{1}{4} a b_{2 ; 2}, \\
G_{34} & =\frac{1}{2} a c_{1 ; 1}+\frac{1}{2} c c_{1 ; 2}-\frac{1}{2} a_{2} b_{1}+\frac{1}{2} c_{1} c_{2}+\frac{1}{2} b c_{2 ; 2}-\frac{1}{4} c a_{1 ; 1}-\frac{1}{4} c b_{2 ; 2}, \\
G_{44} & =\frac{1}{2} a b_{1 ; 1}+c b_{1 ; 2}-\frac{1}{2} c_{1}^{2}+\frac{1}{2} a_{1} b_{1}-\frac{1}{2} b_{1} c_{2}+\frac{1}{2} b_{2} c_{1}+\frac{1}{4} b b_{2 ; 2}-\frac{1}{4} b a_{1 ; 1}-\frac{1}{2} b c_{1 ; 2} .
\end{aligned}
$$

\section{Einstein Walker metrics}

A Walker metric is said to be Einstein Walker metric if its Ricci tensor is a scalar multiple of the metric at each point i.e., there is a constant $\lambda$ so that $\rho=\lambda g$. We have the following result.

Theorem 1. Let $(M, g)$ be a pseudo-Riemannian manifold of dimension 4 , where $g$ is the metric given by (1). Then the following holds: 
(1) If $a=a\left(u_{1}\right), b=b\left(u_{1}\right), c=c\left(u_{1}\right)$, then $(M, g)$ is Einstein if and only if $a=A u_{1}+B ; c=E u_{1}+F$ and $b$ satisfy $a b_{1 ; 1}+a_{1} b_{1}-c_{1}^{2}=0 ;$ where $A, B, E, F$ are constants.

(2) If $a=a\left(u_{1}\right), b=b\left(u_{1}\right), c=c\left(u_{2}\right)$, then $(M, g)$ is Einstein if and only if $a=A u_{1}+B ; b=C u_{1}+D ; c=A u_{1}+F$; where $A, B, C, D, F$ are constants.

(3) If $a=a\left(u_{1}\right), b=b\left(u_{2}\right), c=c\left(u_{2}\right)$, then $(M, g)$ is Einstein if and only if $a=A u_{1}+B ; b=C u_{2}+D$; $c=A u_{2}+F$ where $A, B, C, D, F$ are constants.

(4) If $a=a\left(u_{2}\right), b=b\left(u_{2}\right), c=c\left(u_{2}\right)$, then $(M, g)$ is Einstein if and only if $b=C u_{1}+D ; c=E u_{1}+F$ and $a$ satisfy $b a_{2 ; 2}+b_{2} a_{2}-c_{2}^{2}=0$; where $C, D, E, F$ are constants.

(5) If $a=a\left(u_{1}, u_{2}\right), b=b\left(u_{1}, u_{2}\right)$ and $c=0$ then $(M, g)$ is Einstein if and only if $a=K u_{1}^{2}+A u_{1}+B\left(u_{2}\right)$ and $b=$ $K u_{2}^{2}+C u_{2}+D\left(u_{1}\right) ;$ where $K, A$ and $C$ are constants and $B, D$ are smooth functions satisfying the following PDE's:

$$
\begin{aligned}
B_{2} D_{1} & =0 \\
\left(D_{1}\left(u_{1}^{2} K+u_{1} A+B\right)\right)_{1} & =0 \\
\left(B_{2}\left(u_{2}^{2} K+u_{2} C+D\right)\right)_{2} & =0 .
\end{aligned}
$$

Proof. It follows that the Walker metric (1) is Einstein if and only if the defining functions $a, b$ and $c$ are solutions of the following PDES:

$$
\begin{aligned}
& a_{1 ; 1}-b_{2 ; 2}=0, \quad b_{1 ; 2}+c_{1 ; 1}=0, \quad a_{1 ; 2}+c_{2 ; 2}=0, \\
& a_{1} c_{2}+a_{2} b_{2}-a_{2} c_{1}-c_{2}^{2}+2 c a_{1 ; 2}+b a_{2 ; 2}-a c_{1 ; 2}=0, \\
& a_{2} b_{1}-c_{1} c_{2}+c a_{1 ; 1}-a c_{1 ; 1}-c c_{1 ; 2}+b c_{2 ; 2}=0, \\
& a_{1} b_{1}-b_{1} c_{2}+b_{2} c_{1}-c_{1}^{2}+a b_{1 ; 1}+2 c b_{1 ; 2}-b c_{1 ; 2}=0 .
\end{aligned}
$$

This system of partial differential equations (6) is hard to solve, for this reason we consider the special case in this section. The four first statements are easy to obtain. We only prove the fifth statements. The Einstein condition is equivalent to the following:

(i) $a_{1 ; 2}=0$ and $b_{1 ; 2}=0$;

(ii) $a_{1 ; 1}-b_{2 ; 2}=0$;

(iii) $a_{2} b_{1}=0$;

(iv) $a_{1} b_{1}+a b_{1 ; 1}=0$ and $a_{2} b_{2}+b a_{2 ; 2}=0$.

We divide the proof of the proposition into two steps.

Step 1. The PDE system (i) imply that $a$ and $b$ take the following forms:

$$
a=\bar{a}\left(u_{1}\right)+\hat{a}\left(u_{2}\right) \quad \text { and } \quad b=\bar{b}\left(u_{1}\right)+\hat{b}\left(u_{2}\right) .
$$

Substituting these functions $a$ and $b$ from (7) in the equation (ii), we get

$$
\bar{a}_{1 ; 1}=\hat{b}_{2 ; 2} \text {. }
$$

Therefore we have the following:

$$
\bar{a}_{1 ; 1}(x)=K \quad \text { and } \quad \hat{b}_{2 ; 2}(y)=K
$$

where $K$ is a constant. Then $\bar{a}$ (respectively $\hat{b}$ ) is a quadratic function of $u_{1}$ (respectively $u_{2}$ ). Therefore we have the following

$$
a=K u_{1}^{2}+A u_{1}+B\left(u_{2}\right) \quad b=K u_{2}^{2}+C u_{2}+D\left(u_{1}\right)
$$


where $A$ and $C$ are constants, $B=\hat{a}$ (respectively $D=\bar{b}$ ) are smooth functions of $u_{2}$ (respectively $u_{1}$ ).

Step 2. The functions $a$ and $b$ in (8) satisfy the (i) and (ii) PDEs in the Einstein conditions. We must consider further conditions for $a$ and $b$ to satisfy the (iii) and (iv) PDE in the Einstein condition.

(i) From the (iii) PDE's in the Einstein condition, we get the following condition:

$$
B_{2} D_{1}=0 .
$$

(ii) From (iv), the two equations $a_{1} b_{1}+a b_{1 ; 1}=0$ and $a_{2} b_{2}+b a_{2 ; 2}=0$ gives

$$
\begin{aligned}
& \left(D_{1}\left(u_{1}^{2} K+u_{1} A+B\right)\right)_{1}=0, \\
& \left(B_{2}\left(u_{2}^{2} K+u_{2} C+D\right)\right)_{2}=0,
\end{aligned}
$$

which complete the proof.

In [8], the authors apply the Lie symmetry group method to determine the Lie point symmetry group and provide example of solution of the system of partial differential equations (6).

Example 1. [8] Let $\left(M, g_{a, b, c}\right)$ be a Walker metric with

$a=-\frac{r_{1}}{r_{2}} e^{r_{1} u_{1}} e^{u_{2}}, b=-r_{1} r_{2} e^{r_{1} u_{1}} e^{u_{2}}, \quad$ and $\quad c=r_{2} e^{r_{1} u_{1}} e^{u_{2}}$

where $r_{i}$ 's are arbitrary constants. Then the Walker metric $\left(M, g_{a, b, c}\right)$ is Ricci flat and Einstein.

Note that four-dimensional Einstein Walker manifolds form underling structures of many geometric and physical models such as: $h h$-space in general relativity, $p p$-wave models and others areas.

\section{Locally symmetric Einstein-Walker metrics}

A pseudo-Riemannian manifold is locally symmetric if its curvature tensor $R$ is parallel, that is $\nabla R=0$, where $\nabla$ is the Levi-Civita connection on pseudo-Riemannian extended to act on tensors as a derivation and $R$ is the corresponding curvature tensor. This class of manifolds contains one of manifolds of constant curvature.

Let us consider the Einstein-Walker metric given by

$$
a=K u_{1}^{2}+A u_{1}+B\left(u_{2}\right) \quad \text { and } \quad b=K u_{2}^{2}+C u_{2}+D\left(u_{1}\right),
$$

where $K, A$ and $C$ are constants and $B, D$ are smooth functions satisfying the following PDE's:

$$
\begin{aligned}
B_{2} D_{1} & = \\
\left(D_{1}\left(u_{1}^{2} K+u_{1} A+B\right)\right)_{1} & =0, \\
\left(B_{2}\left(u_{2}^{2} K+u_{2} C+D\right)\right)_{2} & =0 .
\end{aligned}
$$


By a straightforward calculation, we can see that the condition for the Einstein-Walker metric (9) to be locally symmetric is equivalent to the following PDEs

$$
\begin{aligned}
a_{1} a_{2} b_{2} & =0, \quad a_{1} b_{1} b_{2}=0, \quad a_{1} a_{2 ; 2}=0, \quad a_{1} b_{1 ; 1}=0, \\
a_{2} b_{1 ; 1} & =0, \quad b_{1} a_{2 ; 2}=0, \quad b_{2} a_{2 ; 2}=0, \quad b_{2} b_{1 ; 1}=0, \\
a a_{1 ; 1} b_{1} & =0, \quad b a_{2} b_{2 ; 2}=0 .
\end{aligned}
$$

From the previous PDEs, We have the following results

Theorem 2.The Walker metric given in (9) is locally symmetric Einstein if and only if the functions $a\left(u_{1}, u_{2}\right)$ and $b\left(u_{1}, u_{2}\right)$ are constant.

\section{Locally conformally flat Walker metrics}

Let $W$ denote the Weyl conformal curvature tensor given by

$$
\begin{aligned}
W(X, Y, Z, T): & =R(X, Y, Z, T)+\frac{S c}{(n-1)(n-2)}\{g(Y, Z) g(X, T)-g(X, Z) g(Y, T)\} \\
& +\frac{1}{n-2}\{\rho(Y, Z) g(X, T)-\rho(X, Z) g(Y, T)-\rho(Y, T) g(X, Z)+\rho(X, T) g(Y, Z)\} .
\end{aligned}
$$

A pseudo-Riemannian manifold is locally conformally flat if and only if its Weyl tensor vanishes. The nonzero components of Weyl tensor of the Walker metric defined by (1) are given by

$$
\begin{aligned}
& W_{1313}=\frac{a_{1 ; 1}}{6}+\frac{b_{2 ; 2}}{6}-\frac{c_{1 ; 2}}{6}, \quad W_{1314}=-\frac{b_{1 ; 2}}{4}+\frac{c_{1 ; 1}}{4}, \\
& W_{1323}=\frac{a_{1 ; 2}}{4}-\frac{c_{2 ; 2}}{4}, \quad W_{1324}=\frac{c_{1 ; 2}}{2}, \\
& W_{1334}=\frac{c a_{1 ; 1}}{12}-\frac{a b_{1 ; 2}}{4}-\frac{c b_{2 ; 2}}{6}+\frac{5 c c_{1 ; 2}}{12}+\frac{b c_{2 ; 2}}{4} \text {, } \\
& W_{1414}=\frac{b_{1 ; 1}}{2}, \quad W_{1423}=-\frac{a_{1 ; 1}}{12}-\frac{b_{2 ; 2}}{12}+\frac{c_{1 ; 2}}{3}, \quad W_{1424}=\frac{b_{1 ; 2}}{4}-\frac{c_{1 ; 1}}{4} ; \\
& W_{1434}=\frac{b a_{1 ; 1}}{12}+\frac{a b_{1 ; 1}}{4}+\frac{c b_{1 ; 2}}{4}+\frac{b b_{2 ; 2}}{12}-\frac{c c_{1 ; 1}}{4}-\frac{b c_{1 ; 2}}{12}, \\
& W_{2323}=\frac{a_{2 ; 2}}{2}, \quad W_{2324}=-\frac{a_{1 ; 2}}{4}+\frac{c_{2 ; 2}}{4}, \\
& W_{2334}=-\frac{a a_{1 ; 1}}{12}-\frac{c a_{1 ; 2}}{4}-\frac{b a_{2 ; 2}}{4}-\frac{a b_{2 ; 2}}{12}+\frac{a c_{1 ; 2}}{12}-\frac{c c_{2 ; 2}}{4}, \\
& W_{2424}=\frac{a_{1 ; 1}}{6}+\frac{b_{2 ; 2}}{6}-\frac{c_{1 ; 2}}{6}, \quad W_{2434}=\frac{c a_{1 ; 1}}{6}+\frac{b a_{1 ; 2}}{4}-\frac{c b_{2 ; 2}}{12}-\frac{a c_{1 ; 1}}{4}-\frac{5 c c_{1 ; 2}}{12}, \\
& W_{3434}=\frac{c^{2} a_{1 ; 1}}{6}+\frac{a b a_{1 ; 1}}{12}+\frac{b c a_{1 ; 2}}{2}+\frac{b^{2} a_{2 ; 2}}{4}+\frac{a^{2} b_{1 ; 1}}{4}+\frac{a c b_{1 ; 2}}{2}+\frac{c^{2} b_{2 ; 2}}{6}+\frac{a b b_{2 ; 2}}{12} \\
& -\frac{a c c_{1 ; 1}}{2}-\frac{2 c^{2} c_{1 ; 2}}{3}-\frac{a b c_{1 ; 2}}{3}-\frac{b c c_{2 ; 2}}{2}+\frac{b a_{1} c_{2}}{4}-\frac{c a_{1} b_{2}}{4}+\frac{c a_{2} b_{1}}{4}-\frac{b a_{2} c_{1}}{4}-\frac{a b_{1} c_{2}}{4}+\frac{a b_{2} c_{1}}{4} \text {. }
\end{aligned}
$$

Now it is possible to obtain the form of a locally conformally flat Walker metric as follows. 
Theorem 3. A Walker metric (1) is locally conformally flat if and only if the functions $a=a\left(u_{1}, u_{2}\right), b=b\left(u_{1}, u_{2}\right)$ and $c=c\left(u_{1}, u_{2}\right)$ are as follows

$$
\begin{aligned}
& a=\frac{I}{2} u_{1}^{2}+J u_{1}+E u_{1} u_{2}+F u_{2}+K, \\
& b=-\frac{I}{2} u_{2}^{2}+L u_{2}+M u_{1} u_{2}+N u_{1}+R, \\
& c=\frac{M}{2} u_{1}^{2}+P u_{1}+\frac{E}{2} u_{2}^{2}+G u_{2}+(Q+H),
\end{aligned}
$$

where the constants $E, F, G, H, I, J, K, L, M, N, P, Q$ and $R$ satisfy the following relations

$$
\begin{aligned}
& 0=E N-J M+I P, \\
& 0=E L-F M+I G, \\
& 0=E R-K M+I(H+Q), \\
& 0=K(L P-N G)+R(J G-F P)+(Q+H)(F N-J L) .
\end{aligned}
$$

Proof.Sine the locally conformally flat is equivalently to the vanishing of the Weyl tensor, let consider (10) as a system of PDEs. We will prove the theorem in three steps.

Step 1. Considering the following components of the Weyl tensor of (10):

$$
W_{1324}=\frac{c_{1 ; 2}}{2}=0, \quad W_{1414}=\frac{b_{1 ; 1}}{2}=0 \quad \text { and } \quad W_{2323}=\frac{a_{2 ; 2}}{2}=0 .
$$

The PDEs (11) imply that the functions $a, b$ and $c$ take the form

$$
\begin{aligned}
& a\left(u_{1}, u_{2}\right)=u_{2} \bar{a}\left(u_{1}\right)+\hat{a}\left(u_{1}\right), \\
& b\left(u_{1}, u_{2}\right)=u_{1} \bar{b}\left(u_{2}\right)+\hat{b}\left(u_{2}\right), \\
& c\left(u_{1}, u_{2}\right)=\bar{c}\left(u_{1}\right)+\hat{c}\left(u_{2}\right) .
\end{aligned}
$$

Considering the result of the step 1 , the Weyl equations of (10) reduce to

$$
\begin{aligned}
W_{1313}= & \frac{1}{6}\left(a_{1 ; 1}+b_{2 ; 2}\right), \quad W_{1314}=\frac{1}{4}\left(-b_{1 ; 2}+c_{1 ; 1}\right), \quad W_{1323}=\frac{1}{4}\left(a_{1 ; 2}-c_{2 ; 2}\right), \\
W_{1334}= & \frac{c a_{1 ; 1}}{12}-\frac{a b_{1 ; 2}}{4}-\frac{c b_{2 ; 2}}{6}+\frac{b c_{2 ; 2}}{4}, \quad W_{1423}=-\frac{1}{12}\left(a_{1 ; 1}+b_{2 ; 2}\right), \\
W_{1424}= & \frac{1}{4}\left(b_{1 ; 2}-c_{1 ; 1}\right), \quad W_{1434}=\frac{b}{12}\left(a_{1 ; 1}+b_{2 ; 2}\right)+\frac{c}{4}\left(b_{1 ; 2}-c_{1 ; 1}\right), \\
W_{2324}= & \frac{1}{4}\left(-a_{1 ; 2}+c_{2 ; 2}\right), \quad W_{2334}=-\frac{a}{12}\left(a_{1 ; 1}+b_{2 ; 2}\right)-\frac{c}{4}\left(a_{1 ; 2}-c_{2 ; 2}\right), \\
W_{2424}= & \frac{1}{6}\left(a_{1 ; 1}+b_{2 ; 2}\right), \quad W_{2434}=\frac{c a_{1 ; 1}}{6}+\frac{b a_{1 ; 2}}{4}-\frac{c b_{2 ; 2}}{12}-\frac{a c_{1 ; 1}}{4}, \\
W_{3434}= & \frac{c^{2}}{6}\left(a_{1 ; 1}+b_{2 ; 2}\right)+\frac{a b}{12}\left(a_{1 ; 1}+b_{2 ; 2}\right)+\frac{b c}{2}\left(a_{1 ; 2}-c_{2 ; 2}\right)+\frac{a c}{2}\left(b_{1 ; 2}-c_{1 ; 1}\right) \\
& +\frac{b a_{1} c_{2}}{4}-\frac{c a_{1} b_{2}}{4}+\frac{c a_{2} b_{1}}{4}-\frac{b a_{2} c_{1}}{4}-\frac{a b_{1} c_{2}}{4}+\frac{a b_{2} c_{1}}{4} .
\end{aligned}
$$

Step 2. Considering the following components of the PDEs (12):

$$
W_{1313}=0, \quad W_{1314}=0, \quad \text { and } \quad W_{1323}=0 .
$$


The PDEs (13) imply that the functions $a, b$ and $c$ take the form

$$
\begin{aligned}
& a=u_{2}\left(E u_{1}+F\right)+\frac{I}{2} u_{1}^{2}+J u_{1}+K, \\
& b=u_{1}\left(M u_{2}+N\right)-\frac{I}{2} u_{2}^{2}+L u_{2}+R, \\
& c=\frac{M}{2} u_{1}^{2}+P u_{1}+Q+\frac{E}{2} u_{2}^{2}+G u_{2}+H .
\end{aligned}
$$

Considering the result of the step 2, the Weyl equations (12) reduce to

$$
\begin{aligned}
& W_{1334}=\frac{c a_{1 ; 1}}{12}-\frac{a b_{1 ; 2}}{4}-\frac{c b_{2 ; 2}}{6}+\frac{b c_{2 ; 2}}{4}, \\
& W_{2434}=\frac{c a_{1 ; 1}}{6}+\frac{b a_{1 ; 2}}{4}-\frac{c b_{2 ; 2}}{12}-\frac{a c_{1 ; 1}}{4} \\
& W_{3434}=\frac{b a_{1} c_{2}}{4}-\frac{c a_{1} b_{2}}{4}+\frac{c a_{2} b_{1}}{4}-\frac{b a_{2} c_{1}}{4}-\frac{a b_{1} c_{2}}{4}+\frac{a b_{2} c_{1}}{4} .
\end{aligned}
$$

Step 3. From (14), after some straightforward calculations, the following PDES

$$
W_{1334}=0, \quad W_{2434}=0 \quad \text { and } \quad W_{3434}=0
$$

gives

$$
\begin{aligned}
& 0=E N-J M+I P \\
& 0=E L-F M+I G \\
& 0=E R-K M+I(H+Q) \\
& 0=K(L P-N G)+R(J G-F P)+(Q+H)(F N-J L) .
\end{aligned}
$$

This finish the proof.

Remark. From (4) and Theorem 3, we see that the locally conformally flat metric (1) has vanishing scalar curvature.

\section{Conclusion}

Various geometric quantities are computed explicitly in terms of metrics coefficients, including the Christoffel symbols, curvature operator, Ricci curvature and Weyl tensor. Using these formulas, we have obtained a large class of Walker metrics which are Einstein, locally symmetric Einstein and locally conformally flat.

\section{Acknowledgments}

The first author expresses his deepest gratitude for support and hospitality of the University of KwaZulu-Natal.

\section{Competing interests}

The authors declare that they have no competing interests. 


\section{Authors' contributions}

All authors have contributed to all parts of the article. All authors read and approved the final manuscript.

\section{References}

[1] Banyaga A, Massamba F (2016) Non-existence of certain Einstein metrics on some symplectic manifolds, Forum Math 28(3):527537.

[2] Brozos-Vázquez M, García-Rio E, Gilkey P, Nikević S, Vázquez-Lorenzo R (2009) The Geometry of Walker Manifolds. Synthesis Lectures on Mathematics and Statistics, 5. (Morgan and Claypool Publishers, Williston, VT).

[3] Chaichi M, García-Río E, Matsushita Y (2005) Curvature properties of four-dimensional Walker metrics. Classical Quantum Gravity 22:559-577.

[4] García-Río E, Haze S, Katayama N, Matsushita Y (2008) Symplectic, Hermitian and Kahler structures on Walker 4-Manifolds. J. Geom. 90: 56-65.

[5] S. I. Goldberg, Integrability of almost Kahler manifolds, Proc. Amer. Math. Soc. 21 (1969).

[6] Matsushita Y, (2004) Four-dimensional Walker metrics and symplectic structure. J. Geom. Phys. 52: 89-99.

[7] Matsushita Y, (2005) Walker 4-Manifolds with Proper Almost Complex Structure. J. Geom. Phys. 55: 385-398.

[8] Nadjafikhah M, Jafari M (2013) Some general new Einstein Walker manifolds. Adv Math Phys, Art. ID 591852, 8 pp.

[9] K. Sekigawa, On some compact Einstein almost Kahler manifolds, J. Math. Soc. Japan, 39 (1987), nÂ 4, 677-684.

[10] Walker AG (1950) Canonical form for a Riemannian space with a parallel field of null planes. Quart J Math Oxford 1(2): 69-79. 